\title{
LOCATION-BASED SOUND SEGREGATION
}

\author{
Nicoleta Roman, DeLiang Wang \\ Department of Computer and Information Science and Center for \\ Cognitive Science \\ The Ohio State University, Columbus, OH 43210, USA \\ Email: \{niki, dwang\}@ cis.ohio-state.edu
}

\author{
Guy J. Brown \\ Department of Computer Science, University of Sheffield, \\ Sheffield S1 4DP, UK \\ Email: g.brown@dcs.shef.ac.uk
}

\begin{abstract}
We propose and test a novel location-based approach for speech segregation. Within a narrow frequency band modifications to the relative energy of the target source with respect to the interfering energy trigger systematic deviations for binaural cues. For a given spatial configuration, this interaction produces characteristic clustering in the binaural feature space.
\end{abstract}

\section{INTRODUCTION}

The perceptual ability to detect, discriminate and recognize one utterance in a background of acoustic interference has been studied extensively under both monaural and binaural conditions [1] [2] [3]. The auditory system is able to segregate the speech signal from the acoustic mixture using various cues, including pitch, envelope, and location, in a process that is known as auditory scene analysis [1].

It is widely acknowledged that for human audition interaural time differences (ITD) represent the main binaural cue used at low frequencies $(<2 \mathrm{kHz})$, whereas in the high frequency range both interaural intensity differences (IID) and interaural time differences between signal envelopes (IED) are used [2]. The resolution of the binaural cues has implications in both localization and recognition tasks. Experiments show that listeners can reliably detect $10-15 \mathrm{~ms}$ ITD from the median plane, which corresponds to 1-5 degrees in azimuth separation. On the other hand, the smallest detectable change in IID is about $0.5 \mathrm{~dB}$ to $1 \mathrm{~dB}$ at all frequencies. Resolution deteriorates as the reference azimuth gets larger and it has been reported to reach up to 10 degrees when the reference source is located far to the side of the head.

Increased speech intelligibility in binaural listening compared to the monaural case has prompted research in designing cocktail-party processors based on psychoacoustic principles [4] [5] [6]. In particular, building on a previous cross-correlation model for sound localization, Bodden proposed a model that estimates optimal time-varying Wiener coefficients for all critical bands by comparing the neural excitation patterns in cross-correlation with stored patterns obtained from clean speech. Although computationally expensive, Bodden's model can produce substantial enhancement in speech intelligibility.
In this study we propose a sound segregation model using binaural cues extracted from the responses of a KEMAR dummy head that realistically simulates the filtering process of the head and the external ear. We introduce an "ideal" time-frequency binary mask that is motivated by the human auditory masking phenomenon, which selects the target if it is stronger than the interference in a local timefrequency region. If the original unmixed signals are available, one can construct the ideal mask in the following way: retain the time-frequency regions for which target energy exceeds interference energy and discard the other regions. Ideal masks generate high quality reconstruction for a variety of signals, and similar binary masks have been shown to provide a very effective front-end to robust speech recognition [7]. Hence, our model aims to estimate the ideal binary mask. Statistics for the relationship between the relative energy and the deviation of the binaural cues are at the core of our system. We show that for anechoic mixtures of multiple sound sources there exist strong correlation between the energy ratio and ITD and IID cues within a narrow frequency band. For a given spatial configuration, this interaction produces characteristic clustering in the binaural feature space. Consequently, we employ a nonparametric classification method to determine decision regions for the ITD/IID features that correspond to an optimal estimate for the ideal mask.

Related models for estimating target masks have been proposed previously [8] [9]. Such models, however, assume input directly from microphone recordings. As a result, headrelated filtering is not considered. Simulation of human binaural hearing introduces different constraints as well as clues to the problem. First, both ITD and IID should be utilized since IID is more reliable in high frequencies than ITD. Second, frequency-dependent combinations of ITD and IID will arise naturally for a fixed spatial configuration. Consequently, channel-dependent training for each frequency band becomes necessary. Our tests with just ITD (as in [8]) or simple channel-independent classification (as in [9]) yield considerably inferior performance.

Section 2 describes the architecture of our model. Section 3 introduces our method for estimating the ideal binary mask. Section 4 presents systematic evaluation of the system for two and three sources and a comparison with the Bodden model. 


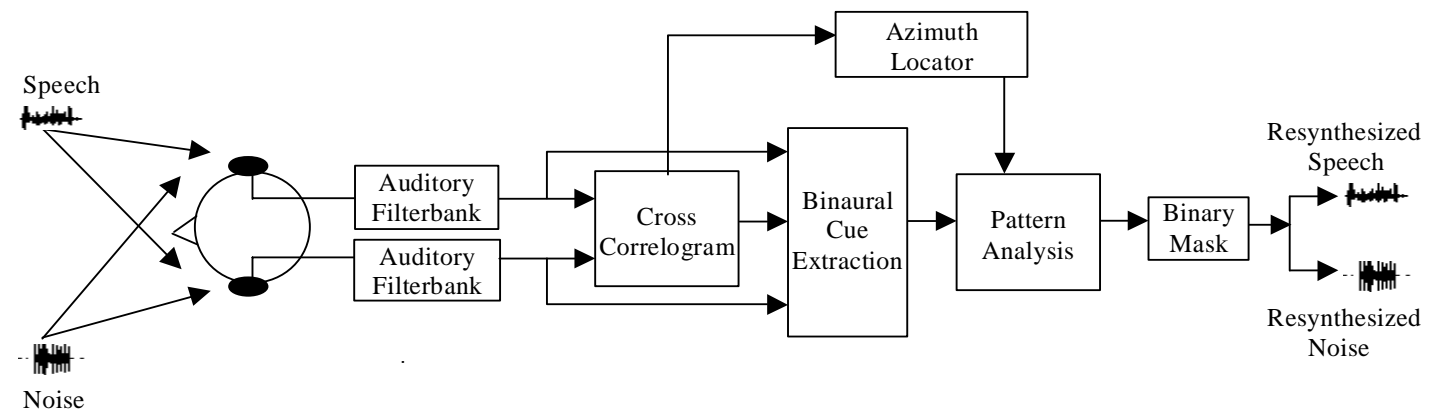

Figure 1: Schematic diagram of the model. We obtain binaural signals by convolving the input signals with head related impulse responses (HRIR). A psychoacoustically motivated auditory periphery is simulated: cochlear filtering, half-wave rectification to simulate auditory nerve firing, low pass filtering for evaluating envelopes at high frequencies and square root to simulate saturation effects. Azimuth localization is based on the cross-correlation mechanism. We compute local ITD and IID independently for all frequency channels. A decision block produces an estimation of the ideal binary mask. The resynthesis path allows the reconstruction of the target signal.

\section{MODEL ARCHITECTURE}

Our model consists of the following five stages: 1) a physiological model of auditory periphery; 2) binaural cue extraction; 3) azimuth localization for both target and interferences; 4) estimation of the ideal binary mask; and 5) resynthesis of the target signal (Fig. 1).

The input to our model is a mixture of two or more signals presented at different, but fixed, locations: target speech and acoustic interferences, which are sampled at 44.1 $\mathrm{kHz}$. Binaural signals are obtained by convolving the input with measured head related impulse responses (HRIR) from a KEMAR dummy head [10].

To simulate the auditory periphery we use a bank of 128 gammatone filters in the range of $80 \mathrm{~Hz}$ to $5 \mathrm{kHz}$ as described in [11]. In addition, the gains of the gammatone filters are adjusted in order to simulate the middle ear transfer function. In the final stage of the peripheral model, the output of each gammatone filter is half-wave rectified in order to simulate the firing probabilities of the auditory nerve. In order to process the envelopes of the signals at higher frequencies a low-pass filter with cutoff frequency of $800 \mathrm{~Hz}$ is considered. Saturation effects are modeled by taking the square root of the signal.

Current models of azimuth localization almost invariably start with the cross-correlation mechanism. We utilize the following azimuth localization method:

1) Compute interaural cross-correlation coefficients at time delays equally distributed in the plausible range from $1 \mathrm{~ms}$ to $1 \mathrm{~ms}$ for all frequency channels.

2) In a training phase, we derive frequency-dependent nonlinear transformations and map the time-delay axis onto an azimuth axis.

3) Calculate an energy-weighted summary across time and frequency and identify the peaks, which generally are very close to the true source locations.

\section{BINARY MASK ESTIMATION}

Our objective is to develop an efficient algorithm for estimating the ideal binary mask. Our estimation is based on the following observation regarding the acoustic interaction of multiple sources: in a narrow band, the ITD and IID corresponding to the target source centers around azimuthdependent characteristic values. As the interference from additional sound sources increases, ITD and IID systematically deviate from these values.

\section{A. Pure Tones}

In order to investigate the relationship between relative signal strengths and ITD for two pure tones with the same frequency $\omega$ we derive the cross-correlation function for the mixture:

$$
\begin{aligned}
c(\tau)= & \frac{A_{1}^{2}}{2} \cos \left(\omega\left(\tau-d_{1}\right)\right)+\frac{A_{2}^{2}}{2} \cos \left(\omega\left(\tau-d_{2}\right)\right)+ \\
& +A_{1} A_{2} \cos \left(\omega\left(\tau-\frac{d_{1}+d_{2}}{2}\right)\right) \cdot \text { coeff }
\end{aligned}
$$

in which $A_{i}$ is the amplitude, $d_{i}$ represents the time delay for the ith source, and $\operatorname{coeff}=\cos \left(\Delta \varphi+\omega\left(d_{2}-d_{1}\right) / 2\right)$ where $\Delta \varphi$ is a function of phase differences between the initial signals and those due to the arrival times of the signals at the left ear. For simplification, IID is considered negligible - true for low-frequency channels. By observing deviation of the peak location $\tau_{\max }$ from the middle location $\left(d_{1}+d_{2}\right) / 2$, we obtain which source is stronger.

On the other hand, we estimate IID as the ratio of energy at the two ears. Therefore, for two pure tones we have: 


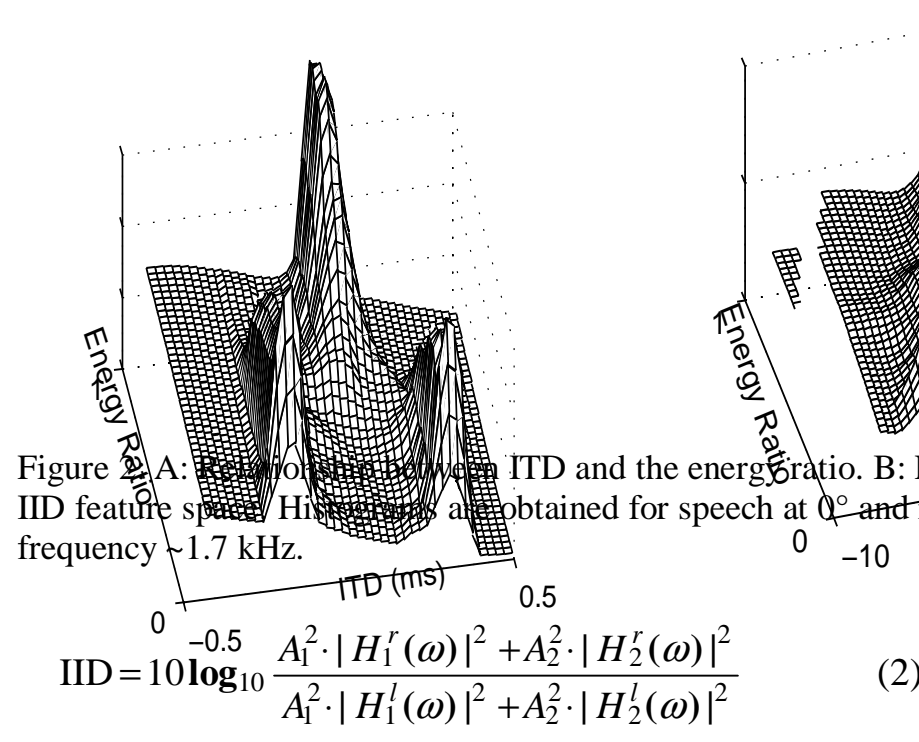

where $H_{i}^{r}(\omega)$ and $H_{i}^{l}(\omega)$ represent the right/left HRTF for the $i$ th source.

A systematic change in the relative amplitude results in a corresponding shift for both ITD and IID. Moreover, thresholds can be derived in order to decide which of the two signals is stronger in a specific region.

\section{B. Real Signals}

Previously, we studied a method of deriving binary decisions independently across frequencies by using ITD for low frequency channels $(<1.5 \mathrm{kHz})$ and IID for high frequency channels $(>1.5 \mathrm{kHz})$ [12]. Here, we consider integrating both features and thus exploit the best discrimination power of the two binaural cues for a specific configuration.

We estimate independently for all frequency channels the local ITD and IID and the energy ratio $E$ based on 20-ms time frames with $10 \mathrm{~ms}$ overlap between adjacent time frames. IID and energy ratios for the $i$ th channel are computed as follows:

$$
\begin{aligned}
& L_{i}=20 \log _{10} \sum_{t} l_{i}^{2}(t) / \sum_{t} r_{i}^{2}(t) \\
& E_{i}=\sum_{t} s_{i}^{2}(t) /\left(\sum_{t} s_{i}^{2}(t)+\sum_{t} n_{i}^{2}(t)\right)
\end{aligned}
$$

where $l_{i}$ and $r_{i}$ refer to the left and right auditory periphery output of the $i$ th channel, respectively; $s_{i}$ refer to the output for the target signal, whereas $n_{i}$ corresponds to the acoustic interference. In computing IID, we use 20 instead of 10 account for the square root operation in the periphery.

In order to eliminate the multiple peaks in the cro correlation function for mid- and high-frequency chann we consider the following strategy. We compute the id ITD for the target source, $l o c_{i}$ for the $i$ th channel. We st deviations from $l o c_{i}$ due to the interferences from ot sources. Consequently, a local ITD is estimated as the de $\tau_{i}^{\max }$ that corresponds to maximum activity in the crc correlation pattern in the range $\left[\operatorname{loc}_{i}-\pi, \operatorname{loc}_{i}+\pi\right]$.

ITD and IID undergo relatively smooth changes n the energy ratio in a given frequency channel. In order capture this relationship, statistics are collected for a gi spatial configuration. We employ the corpus collected Cooke [10], which is commonly used in sound separat studies. The corpus has 100 mixtures obtained from speech utterances mixed with 10 noise intrusic encompassing a variety of common acoustic interferen such as telephone ringing, rock music, and other spee Half of the corpus is used for training, and testing is done the rest of the corpus. In Fig. 2 we display statistics fo channel with center frequency about $1.5 \mathrm{kHz}$ obtained w the target is presented at $0^{\circ}$ (median plane) and two ot sources are active, at $-30^{\circ}$ and $30^{\circ}$. When the information displayed in the ITD-IID plane, we observe location-ba clustering of the binaural cues (Fig. 2C).

Since we are interested in estimating a binary mask, focus on detecting decision regions in the 2-dimensio ITD-IID feature space. Consequently, standard supervi learning techniques can be applied. For the $i$ th channel, test the following two hypotheses. The first one is $I$ target is dominant or $E_{i}>0.5$, and the second one is interference is dominant or $E_{i}<0.5$. Based on estimates the bivariate densities $p\left(x \mid H_{0}\right)$ and $p\left(x \mid H_{1}\right)$ 
classification is done in accordance with the maximum a posteriori decision rule:

$$
\delta(x)= \begin{cases}1, & \text { if } p\left(H_{0}\right) p\left(x \mid H_{0}\right)>p\left(H_{1}\right) p\left(x \mid H_{1}\right) \\ 0, & \text { else }\end{cases}
$$

There exist a plethora of techniques for probability density estimation ranging from parametric techniques (e.g. mixture of gaussians) to nonparametric techniques (e.g. kernel density estimators). In order to completely characterize the distribution of the data we decided to use a kernel density estimation method. The selection of the smoothing parameters is critical to the success of the estimation process: for too small values it approximates the data well but it does not generalize well, for too large values the structure of the data distribution will disappear. One approach for finding the optimal values is the least squares cross validation method [13], which is utilized in our estimation. Optimal values of the parameters are chosen as local minima in the range $\left[1 / 4 n^{-1 / 6} \sigma_{i}, 3 / 2 n^{-1 / 6} \sigma_{i}\right]$ where $\sigma_{i}$ represents the variance for the $i$ th smoothing parameter and $n$ is the sample data number.

\section{EVALUATION}

In order to evaluate the performance of the system for speech segregation, the segregated signal is reconstructed from an estimated binary mask following a resynthesis method described by Brown and Cooke [14]. In order to quantitatively assess system performance, we measure in decibels the SNR using the original speech before mixing as signal:

$$
S N R=10 \log _{10} \sum_{t} s_{o}^{2}(t) / \sum_{t}\left(s_{o}(t)-s_{e}(t)\right)^{2}
$$

where $s_{o}(t)$ represents the resynthesized original speech signal and $s_{e}(t)$ the reconstructed speech from an estimated binary mask signal. One can similarly measure the SNR of the mixture by replacing the denominator with $s_{N}(t)$, the resynthesized original interference.

We compare the SNR gain against results obtained using the ideal binary mask. For two-source segregation, the system is systematically evaluated at the "better ear" for various combinations of azimuth angles. When the target is in the median plane, excellent results are obtained for azimuth separation as small as $5^{\circ}$, as shown in Fig. 3A. Performance degrades when the target source is moved to the side of the head, as shown in Figs. 3B and 3C. This pattern of performance is in agreement with psychoacoustic data [2]. When comparing the SNR with the SNR in the initial mixture, there is an average SNR gain of $14 \mathrm{~dB}$ for target sources in the median plane. This reduces to $11 \mathrm{~dB}$ when the target source is at $70^{\circ}$.
Our approach can be extended to cases with more than two sources. Location methods based on cross-correlation, including ours, are not limited to two locations. With identified locations, our model performs target segregation in a similar manner. Fig. 3D illustrates the performance of our model for the case of three sources with target located in the median plane and two interfering sources at $-30^{\circ}$ and $30^{\circ}$. The average SNR gain obtained is approximately $10 \mathrm{~dB}$. This property of our model differs from many blind source separation and array processing methods where the number of sensors must be no smaller than the number of sources.

In order to draw a quantitative comparison, we have implemented the Bodden model [6], which produces goodquality sound separation using source locations. First, we note that Bodden's cocktail-party processor is a great deal more complicated than ours. His system uses a 24-channel filterbank intended to simulate critical bands and an extended cross-correlation mechanism based on contralateral inhibition in order to compute ITD in the low-frequency range and IID for the high-frequency range. For a fair comparison, our implementation of the Bodden system uses the same 128 channel gammatone filterbank; we also implemented the 24-channel critical bands and the results are not as good. We find that, when two sources are relatively close, the Bodden model is less robust than ours, and our choice of $\left(-10^{\circ}, 30^{\circ}\right)$ falls into the range where his model performs optimally. As shown in Fig. 4, our model shows a considerable improvement over the Bodden system $(\sim 3 \mathrm{~dB})$.

\section{CONCLUSION}

We have presented a location-based sound segregation system, motivated by psychoacoustic and physiological studies of the auditory system. The input to the system is obtained by convolving the original signals with directiondependent HRIRs. The system can be applied to spatial configurations with two or more sources. Our approach is based on an analysis of the relationship between ITD/IID and target/interference energy ratio within narrow bands. Our model yields segregation results which constitute a significant improvement over previous models.

Our study proves that the binaural cues are very effective in filtering out acoustical interference in anechoic room conditions. We are currently extending our model to deal with room reverberations by incorporating the precedence effect and forward/backward masking.

\section{ACKNOWLEDGEMENTS}

This research was supported in part by an NSF grant (IIS-0081058) and an AFOSR grant (F49620-01-0027). 

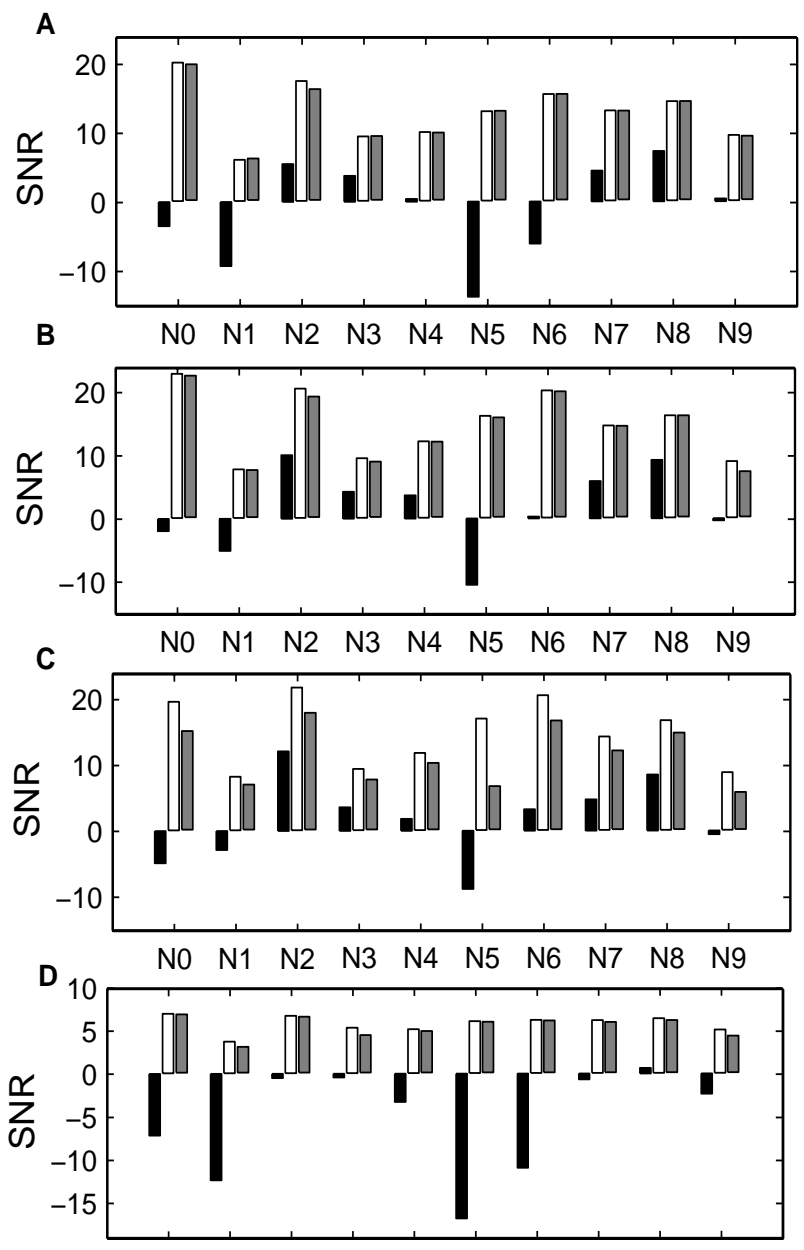

$\begin{array}{llllllllll}\text { N0 } & \mathrm{N} 1 & \mathrm{~N} 2 & \mathrm{~N} 3 & \mathrm{~N} 4 & \mathrm{~N} 5 & \mathrm{~N} 6 & \mathrm{~N} 7 & \mathrm{~N} 8 & \mathrm{~N} 9\end{array}$

Figure 3: Systematic evaluation. Black bar represents the mixture SNR, white bar is the SNR using the ideal binary mask and gray bar corresponds to results from our system. The corpus contains 10 male utterances mixed with ten interferences (N0: pure tone; N1: white noise; N2: noise burst; N3: 'cocktail party'; N4: rock music; N5: siren; N6: trill telephone; N7: female speech; N8: male speech; N9: female speech). A: Target at $0^{\circ}$, interference at $5^{\circ}$. B: Target at $45^{\circ}$, interference at $50^{\circ}$. C: Target at $70^{\circ}$, interference at $75^{\circ}$. D: threesource configuration: target at $0^{\circ}$, interference at $-30^{\circ}$ and $30^{\circ}$.

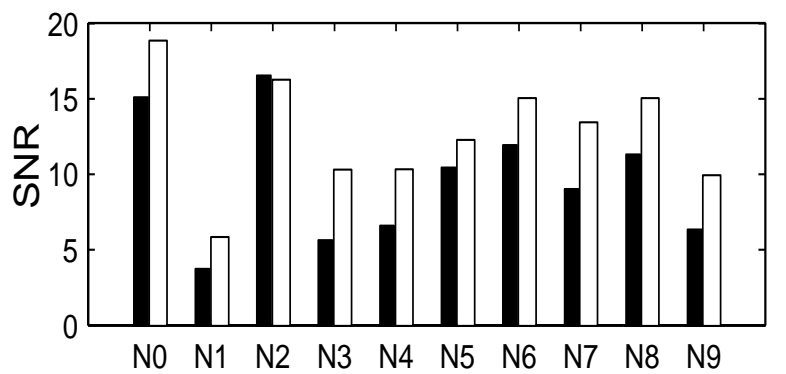

Figure 4: SNR comparison between the Bodden model (black bar) and our model (white bar). Target is at $30^{\circ}$ and interference $-10^{\circ}$.

\section{REFERENCES}

[1] A. S. Bregman, Auditory Scene Analysis, Cambridge, MA: MIT press, 1990.

[2] J. Blauert, Spatial Hearing - The Psychophysics of Human Sound Localization, Cambridge, MA: MIT press, 1997.

[3] A. Bronkhorst, "The cocktail party phenomenon: a review of research on speech intelligibility in multipletalker conditions," Acustica, vol. 86, pp. 117-128, 2000.

[4] R. F. Lyon, "A computational model of binaural localization and separation," Proc. IEEE ICASSP, 1983.

[5] J. Lazarro and C. Mead, "A silicon model for auditory localization," Neural Computation, vol. 1, pp. 47-57, 1989.

[6] M. Bodden, "Modeling human sound-source localization and the cocktail-party-effect," Acta Acoustica, vol. 1, pp. 43-55, 1993.

[7] M.P. Cooke, P. Green, L. Josifovski, and A. Vizinho, "Robust automatic speech recognition with missing and unreliable acoustic data," Speech Comm., vol. 34, pp. 267285, 2001.

[8] H. Glotin, F. Berthommier and E. Tessier, "A CASALabelling model using the localisation cue for, robust cocktail-party speech recognition," Proc. EUROSPEECH, pp.2351-2354, 1999.

[9] A. Jourjine, S. Rickard and O. Yilmaz, "Blind Separation of Disjoint Orthogonal Signals: Demixing N Sources from 2 Mixtures," Proc. IEEE ICASSP, vol. 5, pp. 2985-2988, 2000.

[10] W. G. Gardner and K. D. Martin, "HRTF measurements of a KEMAR dummy-head microphone," MIT Media Lab Perceptual Computing Technical Report \#280, 1994.

[11] D. L. Wang and G. J. Brown, "Separation of speech from interfering sounds based on oscillatory correlation," IEEE Trans. Neural Net., vol. 10, pp. 684-697, 1999.

[12] N. Roman, D. L. Wang and G. J. Brown, "Speech segregation based on sound localization," Proc. IJCNN, pp. 2861-2866, 2001.

[13] B. W. Silverman, Density estimation for statistics and data analysis, New York: Chapman and Hall, 1986.

[14] G. J. Brown and M. P. Cooke, "Computational auditory scene analysis," Computer Speech and Language, vol. 8, pp. 297-336, 1994. 